\title{
Carrier Collective Excitations in Degenerate Semiconductors Studied by EELS
}

\author{
Hongbin Yang, Eric Garfunkel and Philip Batson
}

Rutgers University, Piscataway, New Jersey, United States

Free carriers in semiconductors often dominate their optical and transport properties. Collective excitations of these electrons/holes, due in part to limited dopant solubility, are in the infrared (IR) or terahertz $(\mathrm{THz})$ regime [1]. In the past, experimental studies of semiconductor carrier plasmons were limited to photon-based measurements or reflection electron energy loss spectroscopy (EELS) because of their superior energy resolution [2,3]. Unfortunately, these techniques are limited in spatial resolution.

Here, we present spatially resolved electron energy loss from carrier plasmons in a doped semiconductor, using a Nion UltraSTEM 100, operating at $60 \mathrm{kV}$. As shown in Fig. 1a, spectra were taken from a freestanding indium tin oxide (ITO) film at varying distances from the edge. The spectra taken far away from the edge (1000nm or above) look almost identical. But, if the spectra are taken closer to the edge, the scattering intensities vary significantly due to interference between the surface plasmon polaritons (SPPs) launched by the fast electron, and those reflected by the film edge. The energy loss peak near is due to the bulk carrier plasmon, since at this frequency, . We find that these line-shapes are independent of the grain orientation, as long as they are taken at the same distance from the film edge.

The spatial variations in the scattering intensities are shown in Fig. 2b-d. These spectral images reveal the standing wave patterns of SPP interference with varying wavevector and energy $[4,5]$. The distance from the position of the electron impact to the nearest ITO film edge determines the characteristic wavelength of the excitation. The lower energy SPPs have longer wavelength, and so therefore longer distances between the two maxima. As the energy increases and approaches the surface plasmon energy, $\hbar \omega_{\text {sp }}$, the SPPs have shorter wavelength. The dispersion relation obtained from experimental data clearly shows these excitations originate from the symmetric surface plasmon [6]. In Fig. 2b, energy loss intensities are only observed when the electron beam penetrates the film, characteristic of a bulk mode.

Theoretical EEL probabilities, calculated based on classical dielectric theory [7], provide accurate descriptions of the experimental scattering intensities, allowing us to estimate the carrier concentration and the electronic structure near the surface and grain boundaries of the ITO film. 

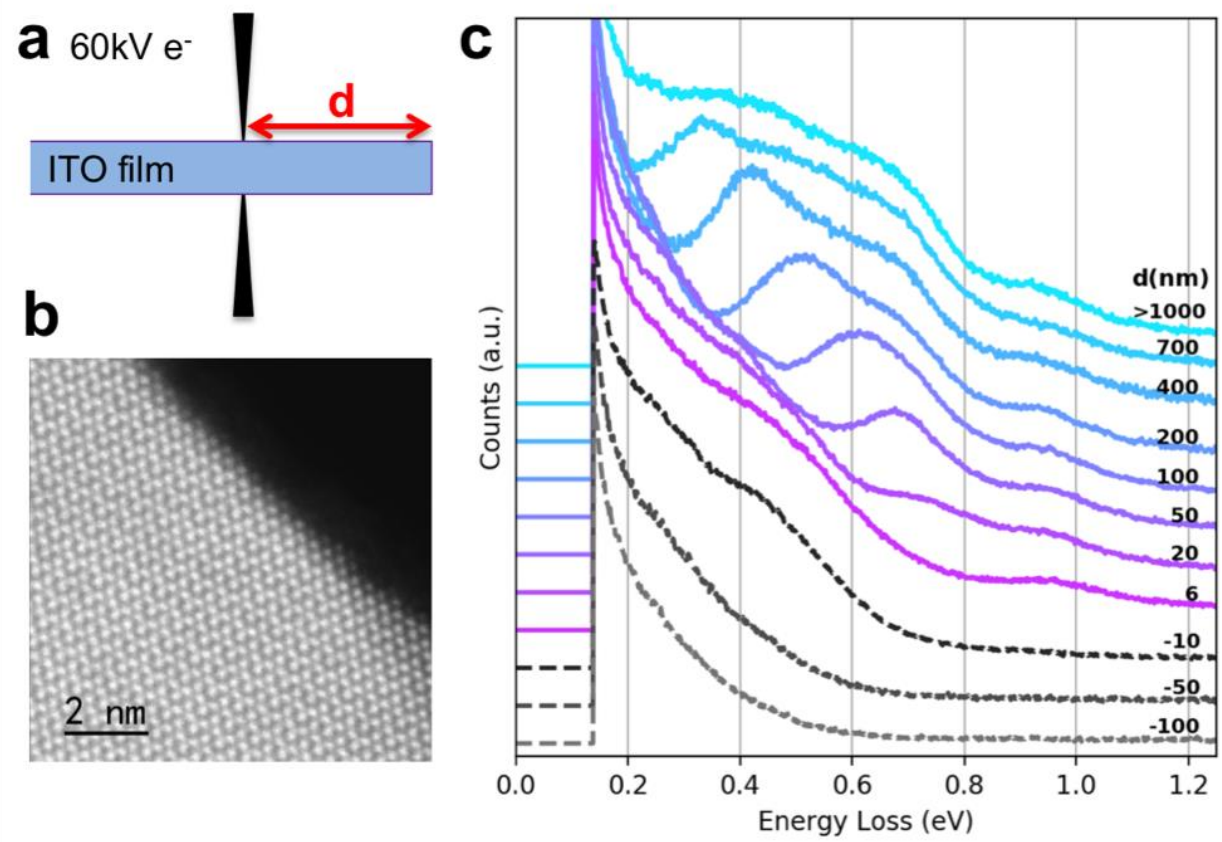

Figure 1. a: Schematic of the energy loss experiment; the red arrow identifies the smallest distance to an ITO edge. b: A high angle annular dark field (HAADF) image for a grain oriented along its [110] axis. c: EELS spectra taken at varying distances to the edge of ITO (solid lines), as well as spectra taken outside the sample edge (dashed lines).

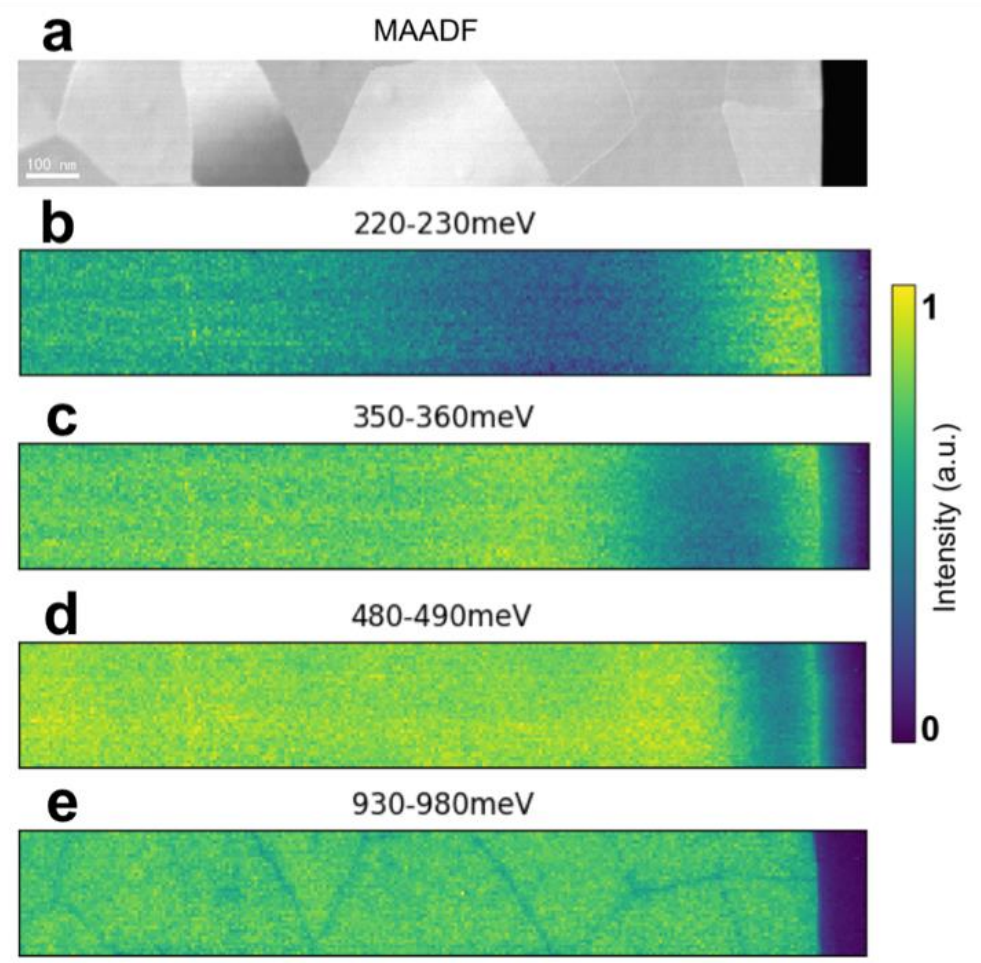

Figure 2. a: A medium angle annular dark field (MAADF) image of the area where the spectrum image is taken. b-d: SPP images generated by integrating spatially resolved spectra within the cited energy range. e: Spectral image of the bulk carrier plasmon. 


\section{References}

[1] J. M. Luther, et al, Nat. Mater., 10, 361-366 (2011)

[2] M. Cardona and G. Guntherodt. Light Scattering in Solid IV. (Springer Berlin, 2014)

[3] H. Lüth. Surface Science 126, 126-146, (1983).

[4] D. Rossouw, et al. Nano Lett. 11, 1499-1504 (2011).

[5] Z. Fei, et al. Nature 487, 82-85 (2012).

[6] R. B. Pettit, J. Silcox, and R. Vincent. Phys. Rev. B 11 (8), 3116 (1975).

[7] A. A. Govyadinov, et al. Nat. Commun. 8, 95 (2017).

H.Y. and P.E.B. acknowledge the financial support of the US Department of Energy, Office of Science, Basic Energy Sciences under award number DE-SC0005132. 